\title{
Tentativa de evidenciar o Trypanosoma cruzi no sangue periférico de pacientes com doença de Chagas, em fase crônica, por meio do Quantitative Buffy Coat (QBC)
}

\author{
An attempt to detect Trypanosoma cruzi in the peripheral blood \\ of patients with Chagas' disease in its chronic phase, \\ by means of Quantitative Buffy Coat (QBC)
}

Vicente Amato Neto, Marta Heloisa Lopes, Cláudia Regina De Marchi e Maria de Fátima dos Santos Silva

\begin{abstract}
Resumo Valorizando a sensibilidade do sistema Quantitative Buffy Coat (QBC), documentada em modelo experimental murino, estando os animais com infecção aguda pelo Trypanosoma cruzi houve tentativa de evidenciar esse parasita no sangue periférico de 100 pacientes com doença de Chagas, em fase crônica. Com o emprego desse método, nenhuma positividade ocorreu, evidentemente em virtude das pequenas parasitemias, não reveláveis pela técnica, pelo menos conforme o verificado através da casuística considerada.
\end{abstract}

Palavras-chaves: Doença de Chagas. Diagnóstico parasitológico. Quantitative Buffy Coat (QBC).

Abstract Taking for garanted the sensivity of the Quantitative Buffy Coat (QBC) system, as documented in a murine esperimental model, we essayed to detect Trypanosoma cruzi in the peripheral blood of 100 patients with Chagas' disease in its chronic phase. By means of the method, no positivity ocurred, evently as a consequence of small parasitemias, undetectable by this technique as assessed by the cases in consideration.

Key-words: Chagas' disease. Parasitologic diagnosis. Quantitative Buffy Coat (QBC).

\footnotetext{
Laboratório de Investigação Médica - Parasitologia do Hospital da Clínicas, da Faculdade de Medicina da Universidade de São Paulo, São Paulo, SP, Brasil.

Endereço para correspondência: Prof. Vicente Amato Neto. Laboratório de Investigação Médica - Parasitologia/FM/USP. Av. Dr. Enéas de Carvalho Aguiar 500, 05403-000 São Paulo, SP, Brasil.

Fax: (011) 852-3622.

Recebido para publicação em 06/08/97.
} 
A fase aguda da doença de Chagas pode apresentar-se através de manifestações clínicas sugestivas e capazes de orientar a efetivação do diagnóstico, sendo que isso tem vínculo com as modalidades congênita ou pós-natal da parasitose. Em determinadas circunstâncias, porém, tais componentes não surgem de maneira claramente aparente, mas em quaisquer situações a definição dessa etapa depende essencialmente da demonstração do Trypanosoma cruzi no sangue periférico por meio de processos diretos, após coloração ou não, com ou sem concentração e enriquecimento.

$\mathrm{Na}$ fase crônica referente a indivíduos que não se encontram sob imunodepressão, motivada por causas diversas, o parasita não é evidenciado quando usados métodos qualificados como diretos e isso não mereceu contestação até o momento, pelo menos quando utilizados os métodos disponíveis e conhecidos.

Amato Neto e cols ${ }^{1}$, recentemente, informaram que, através do sistema Quantitative Buffy Coat (QBC), puderam evidenciar o T. cruzi no sangue de camundongos intencionalmente infectados e estando vigente a fase aguda da parasitose, inclusive após diluição de até 1/10.240. Por isso, consideramos conveniente e oportuno verificar a sensibilidade da técnica em sangue de indivíduos com doença de Chagas, na fase crônica.

Fizeram parte de nossa casuística 100 pacientes, sendo 77 do sexo masculino e 23 do feminino, com idades de 25 a 66 anos. O diagnóstico da doença de Chagas decorreu da positividade das provas sorológicas de fixação do complemento, hemaglutinação passiva e imunofluorescência indireta, envolvendo esta a procura de anticorpos dos tipos $\lg G$ e $\lg M^{2} 5$. As formas clínicas demarcadas foram a indeterminada, a cardíaca de diferentes graus, a digestiva com megaesôfago e a cardíacadigestiva.

Tentamos evidenciar, concomitantemente, o parasita no "creme" leucocitário, obtido em tubo de Wintrobe depois de centrifugação, havendo exame microscópico a fresco. Também realizamos xenodiagnóstico in vitro, com 20 ninfas do Triatoma infestans; como anticoagulante empregamos o citrato de sódio a $3,8 \%$ e levamos em conta o procedimento relatado por Souza e cols 6 .

Retiramos as amostras de sangue para "creme" leucocitário, xenodiagnóstico e QBC no mesmo dia, a propósito de cada doente. Quanto ao QBC, executado em tubo capilar contendo laranja de acridina, sucedendo análise sob luz ultravioleta, no microscópio de fluorescência, com objetiva de 60X e grande profundidade de campo, respeitamos as diretrizes devidamente padronizadas 34 .

Os exames dos "cremes" leucocitários ou efetuados por meio do sistema QBC não revelaram a presença do $T$. cruzi, enquanto houve positividade de cinco xenodiagnósticos (5\%).

A despeito de termos usado método dotado de expressiva sensibilidade, conforme ficou demonstrado em estudo realizado num modelo experimental, calcado na infecção de camundongos, não detectamos, por intermédio dele, o T. cruzi no sangue de pacientes acometidos de doença de Chagas em fase crônica. Com o xenodiagnóstico registramos $5 \%$ de positividades, mas isso é habitual e esperado na citada etapa da parasitose.

Em doentes imunodeprimidos, como decorrência de variados motivos e, presentemente, sobretudo mediante influência do vírus da imunodeficiência humana (HIV), causador da síndrome da imunodeficiência adquirida (AIDS), temos por vezes encontrado o $T$. cruzi no sangue quando usada a técnica que nesta comunicação estamos focalizando. Porém, tais eventos correspondem a reativações parasitárias no período crônico e, portanto, dizem respeito a circunstâncias específicas.

Julgamos que a tentativa realizada e agora relatada era necessária, pois impunha-se verificar o comportamento da capacidade da técnica no estádio crônico da doença de Chagas. Houve insucesso e, talvez, algo diverso aconteça se utilizada casuística bem maior.

O sistema QBC faz parte do conjunto de processos rotulados como diretos e caso tivéssemos evidenciado o protozoário, a caracterização da fase aguda, fundamentada em acontecimento de caráter laboratorial, deveria ser modificada. Entretanto, diante do exposto, não cabe no momento qualquer mudança.

\section{REFERÊNCIAS BIBLIOGRÁFICAS}

1. Amato Neto V, Matsubara L, Lanura PNB. Avaliação do sistema Quantitative Buffy Coat (QBC) no diagnóstico laboratorial da infecção pelo Trypanosoma cruzi: estudo em modelo experimental murino. 
Revista da Sociedade Brasileira de Medicina Tropical 29:59-61, 1996.

2. Camargo ME. Apreciação das técnicas sorológicas atuais para diagnóstico da doença de Chagas. Revista da Sociedade Brasileira de Medicina Tropical 25 (supl III):10, 1992

3. Garin B, Salun JJ, Peyron F, Vigier JP, Busangu I, Perrone J. Rapid in vivo detection of chloroquine resistance by the quantitative buffy coat malaria diagnosis system. The American Journal of Tropical Medicine and Hygiene 47:446-449, 1992.

4. Levine RA, Wardlaw SC, Patton CL. Detection of hematoparasites using quantitative buffy coat analysis tubes. Parasitology Today 5:132-134, 1989.
5. Luquetti AO. Diagnóstico laboratorial da doença de Chagas. Revista da Sociedade Brasileira de Medicina Tropical 25 (supl III): 22-23, 1992.

6. Souza HBW, Moreira AAB, Matsubara L, Campos R, Amato Neto V, Pinto PLS, Takiguti CK. Estudo sobre o xenodiagnóstico "in vitro". II- Comparação com o xenodiagnóstico "in vivo". Revista do Hospital das Clínicas da Faculdade de Medicina de São Paulo 43:165-167, 1988. 